\title{
Effects of serum and serum heat-inactivation on human bone derived osteoblast progenitor cells
}

\author{
A. BRUININK*, U. TOBLER \\ MaTisMed, Biocompatible Materials, Swiss Federal Laboratories for Material Testing and \\ Research (EMPA); Lerchenfeldstrasse 5, CH-9014 St Gallen, Switzerland \\ E-mail: arie.bruinink@empa.ch \\ M. HÄLG \\ FHWädenswil, Grüental, CH-8820 Wädenswil, Switzerland \\ J. GRÜNERT \\ KSSG, Rorschacherstrasse 95, CH-9007 St Gallen, Switzerland
}

Generally, heat inactivated foetal calf serum (FCS) containing media are used for the cultivation of animal and human cells. The role of serum source and serum treatment on the behaviour of cells has long been neglected.

The present study was performed to investigate the effects of serum heat inactivation and serum source on trabecular bone derived progenitor cells (HBC). Furthermore, it was investigated in how far these reactions differed from those seen in bone marrow derived mesenchymal progenitor cells (HBMC) cultures.

We found that HBC cultures performed differently in the presence of FCS and HS with or without heat inactivation. The reactions similar to some degree those observed in HBMC cultures. The implications of the results on cell-implant surface interaction studies are discussed.

(C) 2004 Kluwer Academic Publishers

\begin{abstract}
Introduction
Today knowledge concerning the control and modulation of bone and bone marrow derived osteoblast progenitor cell differentiation is merely based on in vitro studies. In latter studies, in most cases, media are used containing heat-inactivated foetal calf serum (FCS). Heat inactivation is known to reduce haemolytic and anticomplementary activity and to destroy part of the viruses and micro-organisms [1]. Since it has been assumed that it had no or only negligible effects on in vitro cell performance serum heat inactivation has become a common laboratory practice. Recently, we could show that serum source and serum heat inactivation greatly affect bone marrow derived mesenchymal stem cell (HBMC) performance [2]. The HBMC have the potency to differentiate into different cell types like osteoblasts, adipocyte and chondrocytes. Beside HBMCs, it has been suggested that human trabecular bone derived progenitor cell (HBC) cultures are equivalent to mesenchymal stem cell cultures regarding their potential to differentiate into various cell types [3,4]. The HBMC and HBC cells are of importance for the colonisation of bone related implants that should be osseointegrated. We have previously suggested that human serum contains heat labile mitogenic factor(s) that also support osteoblast differentiation. For basic fibroblast growth factor (FGF2) and some other compounds like fluoride it is known

*Author to whom all correspondence should be addressed.
\end{abstract}

that the cell reaction is dependent on the state of cell maturation $[5,6]$. Since cells obtained from the trabecular bone are in a more mature state [7], it may be hypothesized that they react differently on serum source and treatment.

The present study was focused on the influence of heat inactivation and serum choice (human serum (HS) or FCS) on HBC proliferation and differentiation towards osteoblast cells. On one hand, these effects were investigated in the presence of expansion medium, containing FGF-2 and dexamethasone, and on the other hand in osteogenic medium, containing $\beta$-glycerophosphate, ascorbic acid-phosphate, dexamethasone and $1,25-(\mathrm{OH})_{2}$-vitamin $\mathrm{D}_{3}$. The effects found were compared with those found on HBMC.

\section{Materials and methods}

Bone marrow mesenchymal stem cell isolation and expansion

Bone marrow $(5-10 \mathrm{ml})$ of the femur head were obtained from five patients (three males aged 61, 72 and 77 years, and two females aged 53, and 69 years) receiving a total hip prosthesis. To the samples $30 \mathrm{ml}$ ice-cold calciumand magnesium-free isolation medium [8] was added to stop coagulation. Mesenchymal stem cells (HBMC) were isolated from the bone marrow probe by removing soft 
and bone tissue, including fat cells and coagula. Thereafter, cells were filtered through a $200-\mu \mathrm{m}$ mesh filter and collected by centrifugation. The pelleted cells were washed once using isolation medium and finally resuspended in $6 \mathrm{ml} \alpha$-MEM (Gibco, $\mathrm{CH}$ ). One millilitre of this suspension was directly transferred without further purification into $75 \mathrm{~cm}^{2}$ cell culture flasks (TPP AG, CH) containing $19 \mathrm{ml}$ of expansion medium (-E). This medium consist of $90 \% \alpha$-MEM, $10 \%$ serum, $1 \%$ antibiotics (PSN, Gibco, CH), $1 \mathrm{ng} / \mathrm{ml}$ human recombinant fibroblast growth factor type 2 (hrFGF2) and $10 \mathrm{nM}$ dexamethasone. FGF-2 in combination with dexamethasone is known to promote HBMC expansion while keeping the cells osteoblast committed [9]. The sera tested in the present study were FCS (Gibco) without further treatment (FCS), heat inactivated FCS (FCSH), untreated HS and heat inactivated HS (HSH). Heat inactivation was performed at $56^{\circ} \mathrm{C}$ for $30 \mathrm{~min}$. Human $\mathrm{AB}$ rhesus positive blood serum was collected without additives like heparin or citric acid and directly frozen at $-20^{\circ} \mathrm{C}$ after removal of blood cells. Cells were cultured under $95 \%$ humidity, $5 \% \mathrm{CO}_{2}$ and $95 \%$ atmospheric air conditions at $37^{\circ} \mathrm{C}$. After seven days and every third day thereafter medium was replaced by fresh medium after shaking of the cultures. All not firmly attached cells were removed by this step. At confluency of the first flask, cells of all flasks of that experiment were isolated by trypsinization, using $0.25 \%$ trypsin EDTA (Amimed, $\mathrm{CH}$ ). The cells obtained were counted using a coulter counter (Casy Cell Counter) and the yield of every flask was calculated.

\section{Trabecular bone cell isolation and expansion}

To prepare human bone cell (HBC) cultures pieces of femur head trabecular bone of four patients (three males aged 61, 72 and 77 years, and one 72 year old female) were minced and freed from soft tissue, fat and bone marrow by mechanical tools and firm repeated pipetting in analogy as described by Galligher et al. [10]. About $25 \mathrm{mg}$ bone was transferred into $75 \mathrm{~cm}^{2}$ cell culture flasks containing $20 \mathrm{ml}$ of expansion medium containing one of the above mentioned sera. During culture cells were growing out of the bone explants onto the plastic substratum. During the first three weeks in culture medium was replaced by fresh medium once a week and twice a week thereafter. Cells of all flasks were isolated after the first flask of that experiment reached confluency (after around four to six weeks in culture). Thereafter,
HBC cells were isolated by trypsinization and the cell yield per flask was determined. Of every flask, bone pieces were separately collected, dehydrated using ethanol, and vacuum dried. Finally, the weight of the bone pieces was determined.

\section{Cell differentiation and data analysis}

The revealed cells were seeded into wells of 24-well plates in expansion medium containing the serum in which the cells were previously kept. Plating of the cells was defined as the end of passage 0 and start of passage 1 . The cell yield at day 10 after passage was found to be much lower in HBC than in HBMC cultures. Because of that 20000 $\mathrm{HBCs} /$ well and $10000 \mathrm{HBMCs} /$ well were seeded. After three days cultures were washed using $1 \mathrm{ml} \alpha$-MEM without additions. The latter medium was replaced by fresh expansion medium or by osteogenic medium (-D) containing $90 \% \alpha$-MEM, $10 \%$ corresponding serum, $1 \%$ antibiotics, $2 \mathrm{mM} \beta$-glycerophosphate, $0.05 \mathrm{mM}$ ascorbic acid-phosphate, $10 \mathrm{nM}$ dexamethasone and $10 \mathrm{nM}$ 1,25 - $(\mathrm{OH})_{2}$-vitamin $\mathrm{D}_{3}$. Cell performance was determined seven days thereafter by measuring total culture DNA (as index of cell number and by that of cell proliferation), cell protein (as index of cell mass) and alkaline phosphatase (ALP) activity (as index of osteoblast activity) as previously described [11]. The response pattern of the cultures derived from the different donors was similar. Effects of the various media was statistically analysed $(p<0.05$ significance level using two-factorial Bonferroni/Dunn ANOVA taking experiment number and medium type as factors). All results were included. Negative ALP/DNA and protein/DNA data were also included but defined as 0 but latter only if the DNA value was negative (due to blank subtraction). For cell yield comparisons the student $t$-test was used.

\section{Results \\ $\mathrm{HBC}$ cell yield before and after the first passage}

Although bone weight was assessed after the first passage and not on the moment that at start of cultivation bone pieces were added to the flasks no clear-cut weight differences between total amounts added to one or other culture flask containing serum of different source and treatment was found. The cell yield of cells calculated per flask or per mg bone was not affected by heat inactivation of HS or FCS (Table I). Also at the end of the

T A B L E I Effect of serum heat-inactivation and serum source on the yield ratios of HBC cultures kept in expansion medium at the end of the cell expansion, after the first trypsinization (passage 0) and at the end of the experiment (passage 1). Data are presented as mean \pm SEM of 3 or 4 experiments.

\begin{tabular}{|c|c|c|c|c|}
\hline Media compared & $\begin{array}{l}\text { Time point } \\
\text { passage }\end{array}$ & $\begin{array}{l}\text { Cell yield/flask } \\
\text { or culture }(\%)\end{array}$ & $\begin{array}{l}\text { Bone added/mg } \\
\text { to flask }(\%)\end{array}$ & $\begin{array}{l}\text { Cell yield/mg } \\
\text { bone }(\%)\end{array}$ \\
\hline HS-E as $\%$ of HSH-E* & 0 & $127 \pm 30$ & $122 \pm 10$ & $107 \pm 28$ \\
\hline FCS-E as $\%$ of FCSH-E & 0 & $111 \pm 24$ & $103 \pm 4$ & $109 \pm 25$ \\
\hline $\mathrm{HS}-\mathrm{E}$ as $\%$ of $\mathrm{HSH}-\mathrm{E}$ & 1 & $83 \pm 43$ & na & na \\
\hline FCS-E as $\%$ of FCSH-E & 1 & $46 \pm 20$ & na & na \\
\hline
\end{tabular}

na: not applicable; *: HS-E as\% of HSH-E stands for percent value obtained for the cultures kept in HS-E in comparison to values obtained for cultures kept in HSH-E ( $\%$ of HSH-E). 

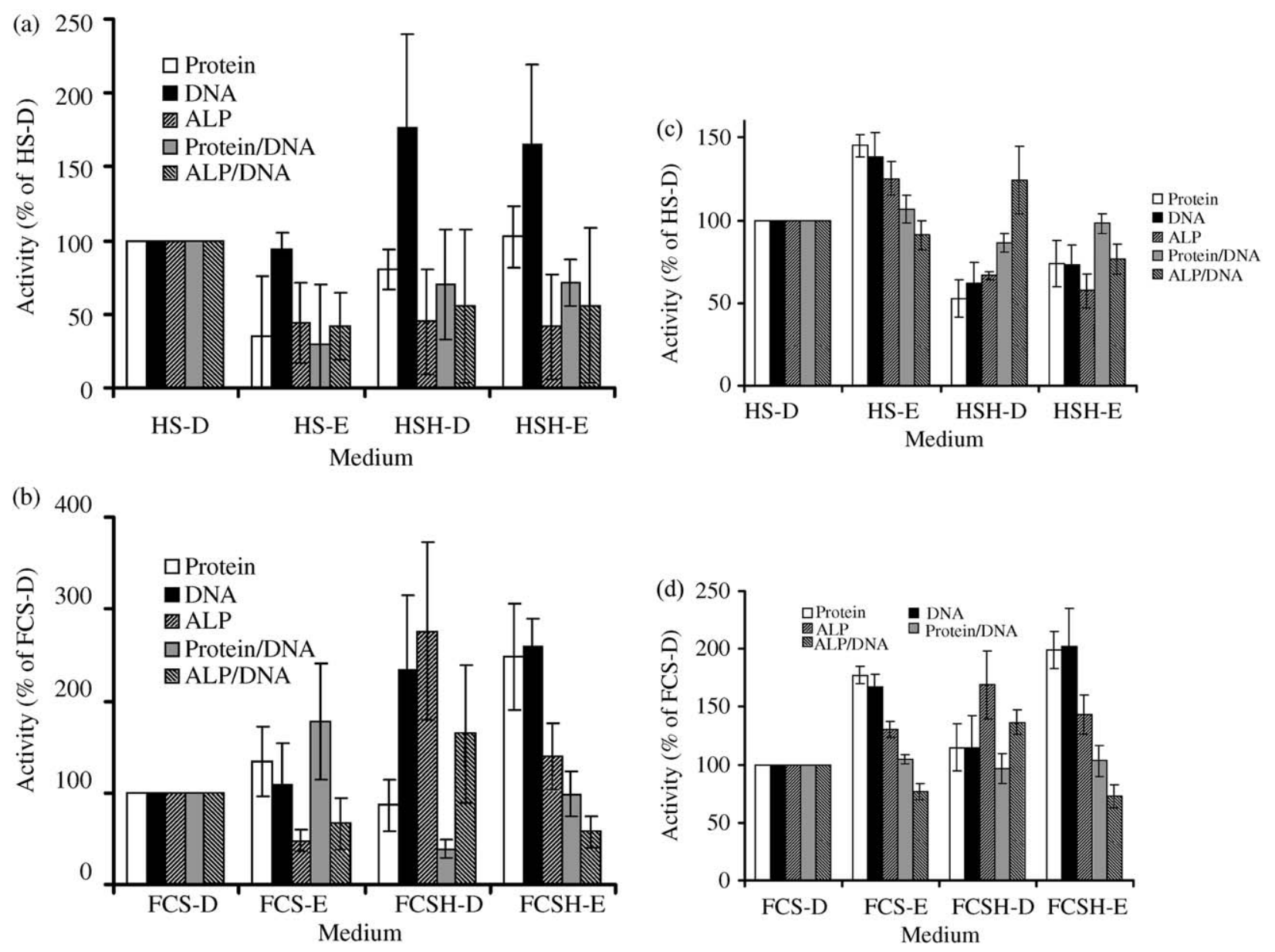

Figure 1 HBC (a, b) and HBMC (c, d) cell performance in the presence of expansion and osteogenic media containing HS (a, c) or FCS (b, d) being heat inactivated or not in comparison to cells cultured in osteogenic medium in the presence of untreated serum for seven days. All cells were kept in expansion medium with the corresponding serum until three days after the first passage after which medium was replaced by osteogenic or expansion medium again containing the corresponding serum. Data are presented as mean \pm SEM of three (HBC) or five experiments (HBMC).

experiment cell yield in HS and HSH and of FCS-E and FCSH-E were similar. However, as concluded from two experiments in which enough bone material from one patient could be collected to investigate in the same experiment effects of HS and FCS (with or without heat inactivation) differences in yield between the sera could be detected. For example, the ratio calculated by dividing the cell yield (calculated as cells/mg bone) at the end of passage 0 in HSH-E by the cell yield in FCSH-E was 0.63 and 0.67 . Similarly, at the end of passage 1 (end of the experiment) the cell yield ratio $\mathrm{HSH}-\mathrm{E} / \mathrm{FCSH}-\mathrm{E}$ was 0.07 and 0.40 , again calculated as cells/mg bone ratio.
Here, it must be noted that both ratio were below 1 although at seeding twice the number of HBCs were present in HSH-E containing cultures in comparison to FCSH-E.

\section{Performance of HBC cells after the first passage}

Heat inactivation of FCS serum increased; in most cases significantly, total culture DNA, cell mass and ALP activity in expansion as well as in osteogenic medium (Fig. 1, Table II(A)). Simultaneously in the presence of

T A B L E I I Summary of all significant differences in $\operatorname{HBC}(\mathrm{A}, \mathrm{B})$ and $\operatorname{HBMC}(\mathrm{C}, \mathrm{D})$ cell performance induced by the FCS (A, C) and HS (B, D) serum and serum treatment (with $(\mathrm{H})$ or without heat inactivation found after one passage and three days in expansion medium plus seven days in expansion (E) or osteogenic (D) medium

\begin{tabular}{|c|c|c|c|c|c|c|c|c|c|c|c|c|}
\hline Medium & FCS-E & & FCS-D & & FCSH-E & & Medium & HS-E & & HS-D & HSH-E & \\
\hline A & & & & & & \multirow{4}{*}{$\downarrow \mathrm{Aa}$} & B & & & & \multirow{4}{*}{\multicolumn{2}{|c|}{ XXXXXXX }} \\
\hline FCSH-D & $\uparrow \mathrm{P}$ & $\downarrow \mathrm{DAa}$ & & $\downarrow \mathrm{DAa}$ & $\uparrow \mathrm{PD}$ & & HSH-D & $\uparrow \mathrm{A}$ & & $\uparrow \mathrm{A}$ & & \\
\hline FCSH-E & & $\downarrow \mathrm{PDA}$ & $\uparrow a$ & $\downarrow \mathrm{PDA}$ & XXXXXXX & & HSH-E & $\uparrow \mathrm{A}$ & $\downarrow \mathrm{P}$ & $\uparrow \mathrm{A}$ & & \\
\hline FCS-D & $\uparrow \mathrm{D}$ & $\downarrow$ Aa & XXXXXXX & & & & HS-D & & $\downarrow \mathrm{A}$ & XXXXXXX & & \\
\hline $\mathrm{C}$ & & & & & & \multirow{4}{*}{$\downarrow$ Aa } & $\mathrm{D}$ & & & & \multirow{4}{*}{$\begin{array}{l}\uparrow \mathrm{PD} \\
\mathrm{XXXXXXX}\end{array}$} & \multirow{4}{*}{$\downarrow \mathrm{Aa}$} \\
\hline FCSH-D & $\uparrow \mathrm{PD}$ & $\downarrow$ Aa & & $\downarrow \mathrm{PAa}$ & $\uparrow \mathrm{PD}$ & & HSH-D & $\uparrow \mathrm{PDA}$ & $\downarrow \mathrm{a}$ & $\uparrow \mathrm{PDAp}$ & & \\
\hline FCSH-E & $\uparrow a$ & $\downarrow \mathrm{PDA}$ & $\uparrow a$ & $\downarrow \mathrm{PDA}$ & XXXXXXX & & HSH-E & $\uparrow \mathrm{PDA}$ & & $\uparrow$ PDAp & & \\
\hline FCS-D & $\uparrow \mathrm{PDA}$ & & XXXXXXX & & & & HS-D & $\uparrow \mathrm{PDA}$ & & XXXXXXX & & \\
\hline
\end{tabular}

P: protein; D: DNA; A: ALP; p: protein/DNA; a: ALP/DNA. The presence of one of these parameter characters within a unit denotes that a significant difference for latter parameter is found ( $p<0.05$, ANOVA Bonferroni/Dunn). Comparisons in which more than one parameter is modified are within a grey unit. Arrows designate the direction of the effects. For example ( $\uparrow$ P) in the unit in Table A second row (FCS-E) and second line (FCSH-D) means that in this case total culture protein values are significantly higher in cultures kept in FCS-E medium in comparison to FCSH-D medium. 
osteogenic medium components, osteoblast cell differentiation (as indicated by ALP activity per DNA) was significantly enhanced.

In analogy to FCS heat-inactivation of HS tends also to promote HBC cell proliferation (Table II(B)). Latter increase was, however, not statistically significant. In contrast to FCS the heat inactivation of HS resulted not in a significant increase, but in a significant decrease of total culture ALP activity, both in expansion and osteogenic medium. Culturing the HBC cells in osteogenic instead of expansion medium resulted in case of FCS and FCSH in decreased total culture DNA and a simultaneous increase in total culture ALP and ALP/DNA values. In this case, the same or at least no contrary effects on cell performance was observed if HS instead of FCS, resp $\mathrm{HSH}$ instead of FCSH was used.

\section{Differences between HBC and HBMC}

Comparing the cell performance in medium containing heat inactivated or untreated FCS in expansion or osteogenic medium, HBMC and HBC behaved very similarly (Table II(A) and (C)). If the reaction of the cultures on the replacement of expansion medium by osteogenic medium was compared in case of untreated FCS, total culture ALP activity in HBC cultures was significantly increased by this replacement and significantly decreased in HBMC. In medium containing heat inactivated FCS, the reaction in HBC and HBMC was not significantly different.

Similarly, HBC and HBMC behaved to some degree in a similar way upon heat inactivation of HS and the use of osteogenic medium instead of expansion medium (Table $\mathrm{II}(\mathrm{B})$ and (D)). Exceptions are that in HBC and HBMC total culture cell protein was significantly affected but in an opposite way by the use of HSH-E medium instead of HS-E medium. Furthermore, by taking osteogenic medium in stead of expansion medium in combination with untreated HS, opposite significant effects on total culture ALP activity in HBC and HBMC cultures were achieved.

\section{Discussion}

One premise of cell culture media is that the cells cultured in this medium behave in vivo-like and that reaction on investigated specific environmental factors is not influenced by the medium composition, i.e. that the reaction is not modified by constituents added to the medium for optimal survival/performance that in vivo normally are not present or not present at the given concentrations. In most cases cells are cultured in the presence of heat inactivated FCS, composed of nutrition's but also of (partly denatured) growth factors and (other) proteins with which the cells in vivo never would come in contact. In the present study evidence was found that serum source and serum treatment indeed was able to modify human cell reactions.

One of the first parameters that are seen as important for most research group in comparing the quality of culture media is its effects on cell survival and proliferation rate. Although some research group found equivalent or enhanced proliferations rates in $\mathrm{HS}$ in comparison to FCS $[12,13]$, others reported that FCS has to be preferred $[14,15]$. Previously, we could show that HBMC cells in HS-E and FCS-E or FCSH-E proliferate at a similar rate before passage 1 and thereafter at comparable rates with maximal values in FCSH-E. In contrast, in HSH-E these cells proliferated at a significant much lower rate during the whole experimental period [2]. In analogy to HBMC, both FCS-E and FCSH-E supported HBC proliferation to the same extent during passage 0 . Thereafter proliferation was in analogy to HBMC found to be slightly enhanced in FCSH-E relative to that in FCS-E. In HS-E and HSH-E, however the cell yield of HBC cells before the first passage was also similar and not significantly markedly reduced as found for HBMC (Table I). In HBC not only cells in $\mathrm{HSH}-\mathrm{E}$ proliferated at a much lower rate but also those kept in HS-E. Our DNA counts at the end of the experiment were in line with latter results. Here, even a tendency of increase proliferation in HSH-E in comparison to HS-E could be seen. Total culture protein exhibited in this respect a similar but significant effect pattern. Part of the differences observed for HBC and HBMC may be traced back to the fact that $\mathrm{HBC}$ are in a more mature state in comparison to HBMC [7]. Maturation-state-dependent differences in reaction has been described for some growth factors like FGF-2 [5]. Growth factor content may be dependent on serum source and be modified by heat inactivation [16]. Thus, our data indicate that the affectivity of HS and FCS to support cell proliferation is dependent on cell source and on heat inactivation of the serum. Latter may explain why different research teams came to a different statement regarding serum source effect on cell proliferation. Furthermore, only by using human serum it could be shown that HBC and HBMC react differently.

Beside cell proliferation, another generally accepted important aspect of a culture medium is its capacity to promote cell differentiation. Others have described that in the presence of expansion components cell proliferation is stimulated while differentiation is inhibited whereas in the presence of osteogenic components cells differentiation towards osteoblasts is stimulated while reducing their capacity to proliferate $[9,17,18]$. In line with latter correlation if instead of expansion medium cultures were kept in osteogenic medium the total culture DNA and total culture cell protein was, or significantly reduced, or at least not significantly affected (Fig. 1, Table II). Latter was in this case independent from the serum used, serum heat inactivation and cells cultured. If heat inactivated serum were used in combination with HBMC and $\mathrm{HBC}$ or untreated serum in combination with HBC simultaneously total culture ALP activity and ALP activity per DNA was significantly increased or at least not significantly affected. These results are also in line with the previous mentioned correlation. A contrasting behaviour is shown by HBMC cultures in the presence of untreated serum (FCS, HS). In later case simultaneous to the osteogenic component induced reduction of total culture protein and DNA also a reduced total culture ALP activity without effect on the degree of osteoblast cell differentiation were detected. These data indicate that HBMC and HBC behaved significantly different in this respect and that these differences only come to light in 
the presence of untreated sera. With other words the experimental statement that could be made was modified only due to serum heat-inactivation.

The clinical success of implants is predominantly determined by their interactions with the surrounding tissue cells. The reaction of cells on implant surfaces is on one hand cell type dependent [19] and on the other hand largely determined by the protein layer covering the implant surface [20]. Latter protein layer is to some degree dependent from the surface characteristics [21] but mainly determined by the composition of the surrounding fluid [22]. Latter layer also plays an important role in in vitro studies. It has been noted that as result of serum heat inactivation, the cell-substratum surface interaction is changed [23]. Our present and previous study [2] indicate that HBMC performance and by that the functional state of the cells is changed by using FCS, FCSH or HSH instead of untreated HS. The simplification of the human in vivo situation towards the in vitro test system has consequences on the predictive value of the results. However, in vitro models not making use of untreated human serum and/or human primary cells of a cell type, that normally, i.e. under in vivo situations, will interact with the investigated implants surfaces, will produce results with a rather undefined and even more limited predictive value.

\section{Acknowledgments}

We acknowledge Dr M. Fopp (RBSD, CH-9007 St Gallen) for the provision of human serum and the whole MaTisMed team, especially Dr Th Osterwalder, for their help and input.

\section{References}

1. R. L. WA R D, J. Clin. Microbiol. 6 (1979) 650.

2. A. BRUININK, M. HÄLG, U. TOBLER and J. GRÜNERT, Eur. J. Cell Biol. (2004) submitted.
3. V. SOTTILE, C. HALLEUX, F. BASSILANA, H. LKELLER and K. SEUWEN, Bone 30 (2002) 699.

4. M. E. nuttall, A. J. Patton, D. L. Olivera, D. P. NADEAU and M. GOWEN, J. Bone Miner. Res. 13 (1998) 371.

5. F. DEBAis, M. HOTT, A. M. GRAULET and P. J. MARIE, ibid. 13 (1998) 645.

6. M. KASSEM, L. MOSEKILDE and E. F. ERIKSEN, Eur. J. Endocrinol. 130 (1994) 381.

7. I. SHUR, F. LOKIEC, I. BLEIBERG and D. BENAYAHU, J. Cell Biochem. 83 (2001) 547.

8. A. BRUININK, in “The Brain in Bits and Pieces", edited by G. Zbinden (MTC Verlag, 1992) p. 23.

9. A. MURAGLiA, I. MARTIN, R. CANCEDdA and R. QUARTO, Bone 22 (1998) 131S.

10. J. A. GALligher, R. GUNDLE and J. N. BERESFORD in "Methods in Molecular Medicine: Human Cell Culture Protocols", edited by G. E. Jones (Hamana Press Inc., 1996) p. 233.

11. A. BRUININK and E. WINTERMANTEL, Biomaterials 22 (2001) 2465.

12. N. YAMAMOTO, M. ISOBE, A. NEGishi, H. YOShimASU, H. SHIMOKAWA, K. OHYA, T. AMAGASA and S. KASUGAI, J. Med. Dent. Sci. 50 (2003) 63.

13. R. O. C. OREFFO, A. S. VIRDI and J. T. TRIFFITT, Eur. J. Cell Biol. 74 (1997) 251.

14. M. G. MCALINDEN and D. J. Wilson, Cell Transplant. 9 (2000) 445.

15. S. A. KUZNETSOV, M. H. MANKANI and P. G. ROBEY, Transplantation 70 (2000) 1780.

16. F. P. HUANG and D. I. S TOT T, Lupus 4 (1995) 297.

17. M. BADDOO, K. HILL, R. WILKINSON, D. GAUPP, C. Hughes, G. C. KOPEN and D. G. Phinney, J. Cell. Biochem. 89 (2003) 1235

18. M. J. COELHO and M. H. FERNANDES, Biomaterials 21 (2000) 1095.

19. P. CLARK, P. CONNOLLY, A. S. G. CURTIS, J. A. T. DOW and C. D. W. WIL KINSON, Development 108 (1990) 635.

20. E. BESS, R. CAVIN, K. MA and J. L. ONG, Implant Dent. 8 (1999) 126.

21. P. B. VAN WACHEM, C. M. VRERIKS, T. BEUGELING, J. FEIJEN, A. BANTJES, J. P. DETMERS and W. G. VAN A KEN, J. Biomed. Mater. Res. 21 (1987) 701.

22. J. G. STEEle, B. A. DALton, G. JOHNSON and P. A. UNDER WOOD, Biomaterials 16 (1995) 1057.

23. D. J. GIAR D, In Vitro Cell. Devel. Biol. 23 (1987) 691.

Received 4 October

and accepted 10 Octerber 2003 